\title{
Intermittency in wind-generated turbulence in Lake Baikal: implications for biophysical couplings in the plankton
}

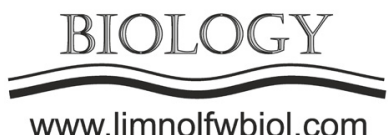

\author{
Seuront L. ${ }^{1,2,3 *}$, Tanaka Y. ${ }^{4}$, Katano T. ${ }^{4,6}$, Sugiyama M. ${ }^{5}$, Watanabe Y. ${ }^{6}$, \\ Shimaraev M.N. ${ }^{7}$, Drucker V.V. ${ }^{7}$
}

${ }^{1}$ The University of Lille, CNRS, Univ. Littoral Côte d'Opale, UMR 8187, LOG, Laboratoire d'Océanologie et de Géosciences, F-59000, Lille, France

${ }^{2}$ Department of Marine Resources and Energy, TUMSAT, Tokyo, Japan

${ }^{3}$ Department of Zoology and Entomology, Rhodes University, Grahamstown, South Africa

${ }^{4}$ Department of Ocean Sciences, TUMSAT, Tokyo, Japan

${ }^{5}$ Department of Natural Resources, Kyoto University, Kyoto, Japan

${ }^{6}$ Faculty of Science, Tokyo Metropolitan University, Tokyo, Japan

${ }^{7}$ Limnological Institute, SB RAS, Irkutsk, Russia

\begin{abstract}
We used tri-hourly wind velocity data recorded near Listvyanka in summer and autumn 1998, 2003, 2005, 2007 and 2011 to estimate the related turbulent kinetic energy dissipation rate $\varepsilon$ affecting the surface waters of Laker Baikal. Though no seasonal nor inter-annual significant differences were found in wind-generated turbulence, clear significant differences were found in their stochastic properties. These properties were subsequently used to assess the effects of intermittent turbulence on critical processes such as nutrient fluxes around phytoplankton cells, coagulation of phytoplankton cells and predator-prey encounter rates.
\end{abstract}

Keywords: Intermittency, turbulence, wind, plankton, biophysical couplings

\section{Introduction}

It is now widely acknowledged that physical and biological patterns and processes observed in a wide range of geographical regions display high intermittency, that is typically characterized by heterogeneous distributions with a few dense patches and a wide range of low-density patches (Fig.). This intermittency may well be a basic property of aquatic ecosystems, as sharp, local fluctuations are ubiquitously observed in space-time distributions of turbulent dissipation rates, temperature, salinity, nutrient and plankton concentration and the motion behaviour of both benthic and pelagic organisms. Critically, through the occurrence of the high-density peaks, intermittency skews a given probability distribution, causing it to deviate from Gaussianity, and as a consequence is hardly compatible with traditional statistical approaches that are typically based on the use of mean turbulence intensity. In this context, we used wind velocity data recorded near Listvyanka in summer and autumn 1998, 2003, 2005, 2007 and 2011 (i) to quantify the intensity of wind-generated turbulence $\varepsilon$ occurring in the surface waters of Lake Baikal, (ii) to assess the properties of intermittent turbulent fluctuations and (iii) to investigate the consequences of intermittent turbulent fluctuations on key processes at the core of the fluxes of matter and energy occurring in the pelagic realm of lake ecosystems.

\section{Methods, Results and Discussion}

First we convert wind velocity into the dissipation rate of the kinetic energy of wind-generated turbulence $\varepsilon\left(\mathrm{m}^{2} \mathrm{~s}^{-3}\right)$ as $\varepsilon=\left(5.82 \times 10^{-9}\right) U^{3} / z$, where $U$ is the wind speed $\left(\mathrm{m} \mathrm{s}^{-1}\right)$ and $z$ the depth (MacKenzie and Leggett, 1993), here fixed as $z=1 \mathrm{~m}$ for the sake of simplicity, and we subsequently infer potential differences in the averaged dissipation rates between months and years. No statistically significant differences $(\mathrm{p}>0.5)$ were found between months and years in $\varepsilon$, which typically ranged between $2.3 \times 10^{-7}$ and $1.8 \times 10^{-6} \mathrm{~m}^{2} \mathrm{~s}^{-3}$, and where typically characterized by their intermittent distributions with a few localized high turbulent patches interspersed over a wide range of low intensity turbulence (Fig.).

A key question that has been previously theoretically investigated in the ocean lies in the understanding of the potential effects of intermittent turbulence, when compared to approaches based on the consideration of averaged turbulence (Seuront, 


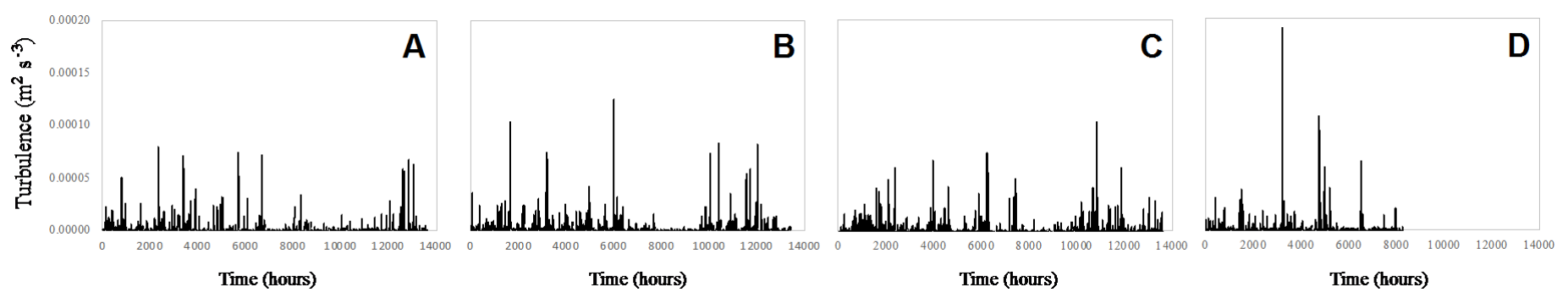

Fig. Illustration of the typical intermittent distributions of wind-generated turbulence observed in the surface waters of Lake Baikal, shown here for (A) August, (B) September, (C) October and (D) November 1998.

2008). In this context, we subsequently assessed the influence on turbulence intermittency on three planktonic processes controlled by turbulent processes, and at the core of aquatic system functioning: nutrient fluxes around non-motile phytoplankton cells, physical coagulation of phytoplankton cells, and predator-prey encounter rates.

We quantified the distribution of the local dissipation rate $\varepsilon_{l}$ as $\left\langle\left(\varepsilon_{l}\right)^{q}\right\rangle=\varepsilon_{0}^{q} \lambda^{K(q)}$, where the angle brackets indicate ensemble averaging, $\lambda$ is the scale ratio between the largest and smallest scales where turbulence exits (i.e. the turbulence inertial subrange), $q$ the statistical order of moment (i.e. $q=1$ and $q=2$ respectively refer to the mean and the variance of the distribution), $\varepsilon_{0}$ the mean turbulence intensity $\left(\varepsilon_{0}^{q}=\left\langle\left(\varepsilon_{l}\right)^{q}\right\rangle\right.$, where $q=1$ ) and $K(q)$ the scaling moment function that characterizes the probability distribution of the fluctuations of $\varepsilon_{r}$. As a consequence, in an intermittent context, in any numerical approach using mathematical functions of the form $f\left(\varepsilon_{0}^{q}\right), \varepsilon_{0}^{q}$ has to be replaced by $\varepsilon_{0}^{q} \lambda^{K(q)}$. We subsequently assessed the influence of intermittent turbulence on key planktonic processes under the influence of turbulence. In short, taking into account the intermittency of turbulence consistently led to a decrease in nutrient fluxes around non-motile phytoplankton cells (15 to $46 \%$ ), physical coagulation of phytoplankton cells (17 to $52 \%$ ) and predator-prey encounter rates (16 to $42 \%$ ).

It is finally stressed that the lack of significant differences in $\varepsilon_{0}$ may erroneously lead to conclude that there is not seasonal nor inter-annual changes in the effect of wind-generated turbulence on Lake Baikal planktonic ecosystems. However, we observed significant changes between years in the function $K(q)$, which leans towards a consistent decrease in the contribution of wind-generated turbulence to nutrient fluxes around non-motile phytoplankton cells, physical coagulation of phytoplankton cells, and predator-prey encounter rates.

\section{Conclusions}

These results show that the intermittent nature of wind-generated turbulence cannot be neglected as it consistently leads to a decrease in the turbulencecontrolled planktonic processes at the core of the structure and function of Lake Baikal pelagic ecosystem. Further work is nevertheless needed to confront our theoretical conclusions to experimental evidence.

\section{References}

MacKenzie B. R. and Leggett W. C. 1993. Wind-based models for estimating the dissipation rates of turbulent energy in aquatic environments: empirical comparisons. Marine Ecology Progress Series 94: 207-216.

Seuront L. 2008. Microscale complexity in the ocean: turbulence, intermittency and plankton life. Mathematical Modelling of Natural Phenomena 3: 1-41. DOI: 10.1051/ mmnp:2008063 